\title{
Erratum to: Porcine P2 myelin protein primary structure and bound fatty acids determined by mass spectrometry
}

\author{
Gianluca Maddalo • Mohammadreza Shariatgorji • \\ Christopher M. Adams • Eva Fung • Ulrika Nilsson • \\ Roman A. Zubarev • Jan Sedzik • Leopold L. Ilag
}

Published online: 5 October 2010

(C) Springer-Verlag 2010

\section{Erratum to: Anal Bioanal Chem \\ DOI 10.1007/s00216-010-3762-0}

Regrettably, there was a mistake in Fig. 1 of this article. The corrected Fig. 1 is given below. In Fig. $2 \mathrm{a}$ the label in the spectrum should correctly read $[\mathrm{M}+3 \mathrm{H}]^{3+}$. In the electronic supplementary material, in Fig. S2, the label in the spectrum should correctly read $[\mathrm{M}+2 \mathrm{H}]^{2+}$.

The online version of the original article can be found at http://dx.doi. org/10.1007/s00216-010-3762-0.

G. Maddalo $\cdot$ M. Shariatgorji $\cdot$ U. Nilsson $\cdot$ L. L. Ilag $(\bowtie)$

Department of Analytical Chemistry, Stockholm University,

Svante Arrhenius väg 16,

10691 Stockholm, Sweden

e-mail: leopold.ilag@anchem.su.se

C. M. Adams $\cdot$ E. Fung $\cdot$ R. A. Zubarev

Institute for Cell and Molecular Biology, Uppsala University,

75124 Uppsala, Sweden

C. M. Adams $\cdot$ E. Fung $\cdot$ R. A. Zubarev

Division of Molecular Biometry,

Institute for Medicinal Biochemistry and Biophysics,

Karolinska Institutet,

Scheeles väg 2,

17177 Stockholm, Sweden

\section{J. Sedzik}

Department of Neurobiology, Health Care and Society,

Protein Crystallization Facility, Karolinska Institute, NOVUM,

14186 Stockholm, Sweden

J. Sedzik

Department of Chemical Engineering and Technology,

Protein Crystallization Facility,

Royal Institute of Technology, KTH,

Teknikringen 28,

10044 Stockholm, Sweden

\section{J. Sedzik}

Division of Neurobiology and Bioinformatic,

National Institute of Physiological Science,

Nishigonaka Myadaiji,

Okazaki 444-8585 Aichi, Japan

\section{J. Sedzik}

Department of Applied Chemistry, School of Engineering 7-3-1,

The University of Tokyo,

Hongo, Bunkyo 113-8656 Tokyo, Japan 


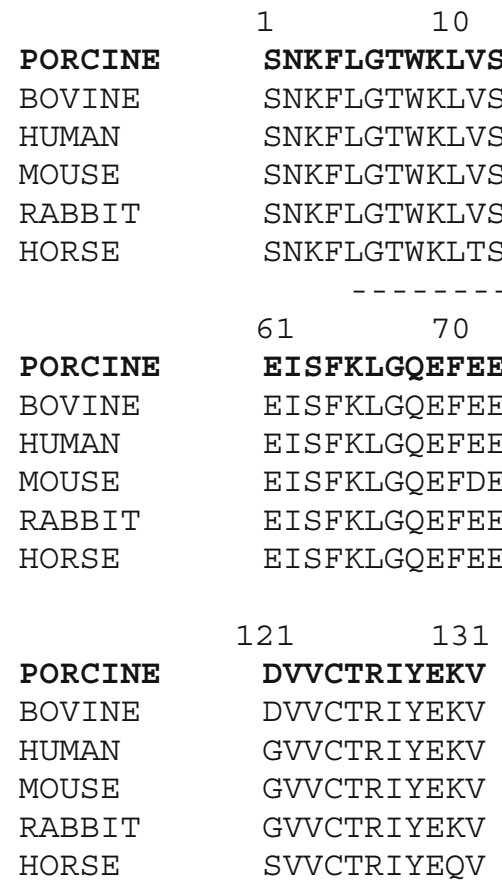

Fig. 1 Multiple sequence alignment of porcine myelin P2 protein with homologs from five other species. The porcine sequence is indicated in bold. Highlighted in boxes are the amino acid substitutions between porcine and bovine sequences determined in this study. The lipid-binding domain as defined by the software ScanProsite [40] is underlined 